\title{
Theoretical Models for Magnetic Properties of Iron Pnictides Part II: Boson Formalism
}

\author{
M. Vujinović, M. Pantić, M. Pavkov-Hrvojević, P. Mali \\ University of Novi Sad, Faculty of Sciences, Department of Physics, \\ Trg Dositeja Obradovića 4, 21000 Novi Sad, Serbia
}

Received: December 16, 2011

\begin{abstract}
We analyze the $J_{1}-J_{2}-J_{c}$ Heisenberg model Hamiltonian by using the DysonMaleev representation for spin operators and keeping the terms quadratic in Bose operators (linear spin wave theory). From the resulting Hamiltonian we find the ground state magnetisation and spin wave dispersion by employing the Green's function method. We compare the results with those of random phase approximation analysis from Part I and with experimental data for parent pnictide compounds. Neither of the two approaches gives a completely satisfactory description of the magnetic properties of iron pnictides. We conclude that alterations of the model Hamiltonian are needed to get a better agreement between the theory and experiments.
\end{abstract}

Key words: Iron pnictides, Green's functions, linear spin wave

\section{Introduction}

In our accompanying paper [1] (referred to as Part I) we introduced the $J_{1}-J_{2}-J_{c}$ Heisenberg Hamiltonian and obtained the ground state magnetization and dispersion law for this Hamiltonian. The expressions for magnetization and magnon dispersion were analyzed numerically and the applicability of the model to undoped iron pnictides was checked by comparison with experimental evidence. The theoretical results do not completely agree with experimental ones, meaning that the model needs to be modified to make it more relevant to parent pnictide compounds. However, before we haste into any alteration of the model we would like to derive the magnetization and dispersion law using a different approach.

In Part I we analyzed the model Hamiltonian by using the Green's function method $[2,3,4]$ for spin operators. Spin formalism is powerful, but it has a few shortcomings. Spin operators obey commutation relations which are fairly complicated. Also, it is well known that the Fourier tranformation is not a canonical transformation for spin operators. This is why, when going into momentum space, we introduced the Fourier transformation for the Green's function (eq. (4) of Part I), and not for each operator separately. Finally, in order to decouple the equations of motion for Green's functions we had to employ the random phase approximation (RPA), and the influence of this approximation on the 
results is very hard to evaluate. It is therefore desirable for us to obtain the magnetization and the dispersion law using a different method, as a check on the results we got using the RPA approach. Our second approach will be based on the Dyson-Maleev representation $[5,6]$ for spin operators. For easier reference, we will write down the model Hamiltonian once more:

$$
\hat{H}=J_{1} \sum_{\langle\mathbf{i}, \mathbf{j}\rangle} \mathbf{S}_{\mathbf{i}} \cdot \mathbf{S}_{\mathbf{j}}+J_{2} \sum_{\langle\langle\mathbf{i}, \mathbf{j}\rangle\rangle} \mathbf{S}_{\mathbf{i}} \cdot \mathbf{S}_{\mathbf{j}}+J_{c} \sum_{|\mathbf{i}, \mathbf{j}|} \mathbf{S}_{\mathbf{i}} \cdot \mathbf{S}_{\mathbf{j}}
$$

We remind the reader that because of the layered structure of iron pnictides [7], the NN interplane exchange integral $J_{c}$ will be considered to be at least one order of magnutude smaller than the NN intraplane exchange integral $J_{1}$.

\section{Bosonization and the LSW approach}

Most alternatives to spin formalism are based on the substitution of spin operators with Bose operators. The biggest shortcoming of all these methods is the fact that spin operators $\hat{S}^{+}$and $\hat{S}^{-}$can create or anihillate only a finite number of excitations, while Bose operators can create or anihillate any number of excitations from zero to infinity. This is why the Bose operators can be considered to be a good substitution for spin operators only when the average number of excitations is smaller or equal to $2 S$, with $S$ being the spin quantum number. In other words, the boson formalism works best for systems with large spin quantum numbers and for low temperatures. The systems which we consider in this paper do not possess a high spin quantum number (we mentioned in Part I that $S=1$ seems to be a relevant choice for iron pnictides [8]), but we are only interested in the results for absolute zero temperature, so we expect that the boson formalism will produce reliable results.

There is more than one way to bosonize the spin Hamiltonian. We will use the DysonMaleev representation for spin operators. In this representation the spin operators for sublattices $a$ and $B$ are given in terms of the corresponding Bose-operators as:

$$
\begin{aligned}
\hat{S}_{i}^{z(a)} & =S-\hat{a}_{i}^{\dagger} \hat{a}_{i} & \hat{S}_{j}^{z(B)} & =S-\hat{B}_{j}^{\dagger} \hat{B}_{j} \\
\hat{S}_{i}^{+(a)} & =\sqrt{2 S} \hat{a}_{i} & \hat{S}_{j}^{+(B)} & =\sqrt{2 S} \hat{B}_{j} \\
\hat{S}_{i}^{-(a)} & =\sqrt{2 S}\left(\hat{a}_{i}^{\dagger}-\frac{1}{2 S} \hat{a}_{i}^{\dagger} \hat{a}_{i}^{\dagger} \hat{a}_{i}\right) & \hat{S}_{j}^{-(B)} & =\sqrt{2 S}\left(\hat{B}_{j}^{\dagger}-\frac{1}{2 S} \hat{B}_{j}^{\dagger} \hat{B}_{j}^{\dagger} \hat{B}_{j}\right)
\end{aligned}
$$

For the sublattices $A$ and $b$, similar expressions hold:

$$
\begin{aligned}
\hat{S}_{i}^{z(b)} & =S-\hat{b}_{i}^{\dagger} \hat{b}_{i} & \hat{S}_{j}^{z(A)} & =S-\hat{A}_{j}^{\dagger} \hat{A}_{j} \\
\hat{S}_{i}^{-(b)} & =\sqrt{2 S} \hat{b}_{i}^{\dagger} & \hat{S}_{j}^{-(A)} & =\sqrt{2 S} \hat{A}_{j}^{\dagger} \\
\hat{S}_{i}^{+(b)} & =\sqrt{2 S}\left(\hat{b}_{i}-\frac{1}{2 S} \hat{b}_{i}^{\dagger} \hat{b}_{i} \hat{b}_{i}\right) & \hat{S}_{j}^{+(A)} & =\sqrt{2 S}\left(\hat{A}_{j}-\frac{1}{2 S} \hat{A}_{j}^{\dagger} \hat{A}_{j} \hat{A}_{j}\right)
\end{aligned}
$$

For simplicity, $i$ and $j$ denote the lattice site vectors. It is obvious that in the DM representation spin operators $S^{+}$and $S^{-}$are not hermitian conjugates of each other, and thus the Hamiltonian is not a hermitian operator. More precisely, in the DM representation the Hamiltonian takes the form:

$$
\hat{H}=\hat{H}_{0}+\hat{H}_{2}+\hat{H}_{4}
$$


Here $\hat{H}_{0}$ is a constant, $\hat{H}_{2}$ contains the terms quadratic in Bose-operators, while $\hat{H}_{4}$ contains terms quartic in Bose-operators. The $\hat{H}_{4}$ term is a non-hermitian operator. However, we will keep only the $\hat{H}_{0}$ and $\hat{H}_{2}$ terms, which corresponds to Bloch's approximation, or linear spin wave (LSW) theory. After the bosonization and Bloch's approximation the model Hamiltonian (1) becomes:

$$
\begin{aligned}
& \hat{H}=E_{0}+ \\
& \quad+S J_{1} \sum_{i, \delta_{x}}\left(\hat{A}_{i}^{\dagger} \hat{A}_{i}+\hat{a}_{i+\delta_{x}}^{\dagger} \hat{a}_{i+\delta_{x}}+\hat{A}_{i} \hat{a}_{i+\delta_{x}}+\hat{A}_{i}^{\dagger} \hat{a}_{i+\delta_{x}}^{\dagger}+\hat{B}_{i}^{\dagger} \hat{B}_{i}+\hat{b}_{i+\delta_{x}}^{\dagger} \hat{b}_{i+\delta_{x}}+\hat{B}_{i} \hat{b}_{i+\delta_{x}}+\hat{B}_{i}^{\dagger} \hat{b}_{i+\delta_{x}}^{\dagger}\right)- \\
& \quad-S J_{1}^{F} \sum_{i, \delta_{y}}\left(\hat{A}_{i}^{\dagger} \hat{A}_{i}+\hat{b}_{i+\delta_{y}}^{\dagger} \hat{b}_{i+\delta_{y}}-\hat{A}_{i} \hat{b}_{i+\delta_{y}}^{\dagger}-\hat{A}_{i}^{\dagger} \hat{b}_{i+\delta_{y}}+\hat{a}_{i}^{\dagger} \hat{a}_{i}+\hat{B}_{i+\delta_{y}}^{\dagger} \hat{B}_{i+\delta_{y}}-\hat{a}_{i} \hat{B}_{i+\delta_{y}}^{\dagger}-\hat{a}_{i}^{\dagger} \hat{B}_{i+\delta_{y}}\right)+ \\
& \quad+S J_{c} \sum_{i, \delta_{z}}\left(\hat{a}_{i}^{\dagger} \hat{a}_{i}+\hat{b}_{i+\delta_{z}}^{\dagger} \hat{b}_{i+\delta_{z}}+\hat{a}_{i} \hat{b}_{i+\delta_{z}}+\hat{a}_{i}^{\dagger} \hat{b}_{i+\delta_{z}}^{\dagger}+\hat{A}_{i}^{\dagger} \hat{A}+\hat{B}_{i+\delta_{z}}^{\dagger} \hat{B}_{i+\delta_{z}}+\hat{A}_{i} \hat{B}_{i+\delta_{z}}+\hat{A}_{i}^{\dagger} \hat{B}_{i+\delta_{z}}^{\dagger}\right)+(9) \\
& \quad+S J_{2} \sum_{i, \delta_{2}}\left(\hat{a}_{i}^{\dagger} \hat{a}_{i}+\hat{b}_{i+\delta_{2}}^{\dagger} \hat{b}_{i+\delta_{2}}+\hat{a}_{i} \hat{b}_{i+\delta_{2}}+\hat{a}_{i}^{\dagger} \hat{b}_{i+\delta_{2}}^{\dagger}+\hat{A}_{i}^{\dagger} \hat{A}_{i}+\hat{B}_{i+\delta_{2}}^{\dagger} \hat{B}_{i+\delta_{2}}+\hat{A}_{i} \hat{B}_{i+\delta_{2}}+\hat{a}_{i}^{\dagger} \hat{b}_{i+\delta_{2}}^{\dagger}\right)
\end{aligned}
$$

Once we bosonize the Hamiltonian in this way, we go into momentum space via Fourier transformation. Since the Fourier transformation is canonical for Bose operators, we may introduce the transformation for each operator separately. For operators in the sublattice $a$ this transformation takes the form:

$$
\hat{a}_{n}=\frac{1}{\sqrt{N}} \sum_{k} \hat{a}_{k} e^{+i k \cdot n} \quad \hat{a}_{n}^{\dagger}=\frac{1}{\sqrt{N}} \sum_{k} \hat{a}_{k}^{\dagger} e^{-i k \cdot n}
$$

And similarly for other sublattices. After the Fourier transformation the LSW Hamiltonian becomes:

$$
\begin{aligned}
\hat{H} & =E_{0}+ \\
& +2 S J_{1} \sum_{k}\left(\hat{O}_{k}+\cos a k_{x}\left(\hat{A}_{k} \hat{a}_{-k}+\hat{A}_{k}^{\dagger} \hat{a}_{-k}^{\dagger}+\hat{B}_{k} \hat{b}_{-k}+\hat{B}_{k}^{\dagger} \hat{b}_{-k}^{\dagger}\right)\right)- \\
& -2 S J_{1}^{F} \sum_{k}\left(\hat{O}_{k}-\cos a k_{y}\left(\hat{A}_{k} \hat{b}_{k}^{\dagger}+\hat{A}_{k}^{\dagger} \hat{b}_{k}+\hat{a}_{k} \hat{B}_{k}^{\dagger}+\hat{a}_{k}^{\dagger} \hat{B}_{k}\right)\right)+ \\
& +4 S J_{2} \sum_{k}\left(\hat{O}_{k}+\cos a k_{x} \cos a k_{y}\left(\hat{A}_{k} \hat{B}_{-k}+\hat{A}_{k}^{\dagger} \hat{B}_{-k}^{\dagger}+\hat{a}_{k} \hat{b}_{-k}+\hat{a}_{k}^{\dagger} \hat{b}_{-k}^{\dagger}\right)\right)+ \\
& +2 S J_{c} \sum_{k}\left(\hat{O}_{k}+\cos c k_{z}\left(\hat{A}_{k} \hat{B}_{-k}+\hat{A}_{k}^{\dagger} \hat{B}_{-k}^{\dagger}+\hat{a}_{k} \hat{b}_{-k}+\hat{a}_{k}^{\dagger} \hat{b}_{-k}^{\dagger}\right)\right)
\end{aligned}
$$

The $\hat{O}_{k}$ operator contains the diagonal terms:

$$
\hat{O}_{k}=\hat{A}_{k}^{\dagger} \hat{A}_{k}+\hat{a}_{k}^{\dagger} \hat{a}_{k}+\hat{B}_{k}^{\dagger} \hat{B}_{k}+\hat{b}_{k}^{\dagger} \hat{b}_{k}
$$

Now that we have the model Hamiltonian in momentum space, we can find the equations of motion for Green's functions which contain the operators that create excitations in their respective sublattices. These equations are:

$$
\begin{aligned}
(\omega-\varepsilon)\left\langle\left\langle\hat{A}_{k} \mid \hat{R}\right\rangle\right\rangle_{\omega}-J_{x}\left\langle\left\langle\hat{a}_{-k}^{\dagger} \mid \hat{R}\right\rangle\right\rangle_{\omega}-J_{y}\left\langle\left\langle\hat{b}_{k} \mid \hat{R}\right\rangle\right\rangle_{\omega}-J_{2 c}\left\langle\left\langle\hat{B}_{-k}^{\dagger} \mid \hat{R}\right\rangle\right\rangle_{\omega} & =\frac{i}{2 \pi}\left\langle\left[\hat{A}_{k}, \hat{R}\right]\right\rangle \\
J_{x}\left\langle\left\langle\hat{A}_{k} \mid \hat{R}\right\rangle\right\rangle_{\omega}+(\omega+\varepsilon)\left\langle\left\langle\hat{a}_{-k}^{\dagger} \mid \hat{R}\right\rangle\right\rangle_{\omega}+J_{y}\left\langle\left\langle\hat{b}_{k} \mid \hat{R}\right\rangle\right\rangle_{\omega}-J_{2 c}\left\langle\left\langle\hat{B}_{-k}^{\dagger} \mid \hat{R}\right\rangle\right\rangle_{\omega} & =\frac{i}{2 \pi}\left\langle\left[\hat{a}_{-k}^{\dagger}, \hat{R}\right]\right\rangle \\
-J_{y}\left\langle\left\langle\hat{A}_{k} \mid \hat{R}\right\rangle\right\rangle_{\omega}-J_{2 c}\left\langle\left\langle\hat{a}_{-k}^{\dagger} \mid \hat{R}\right\rangle\right\rangle_{\omega}+(\omega-\varepsilon)\left\langle\left\langle\hat{b}_{k} \mid \hat{R}\right\rangle\right\rangle_{\omega}-J_{x}\left\langle\left\langle\hat{B}_{-k}^{\dagger} \mid \hat{R}\right\rangle\right\rangle_{\omega} & =\frac{i}{2 \pi}\left\langle\left[\hat{b}_{k}, \hat{R}\right]\right\rangle
\end{aligned}
$$




$$
J_{2 c}\left\langle\left\langle\hat{A}_{k} \mid \hat{R}\right\rangle\right\rangle_{\omega}+J_{y}\left\langle\left\langle\hat{a}_{-k}^{\dagger} \mid \hat{R}\right\rangle\right\rangle_{\omega}+J_{x}\left\langle\left\langle\hat{b}_{k} \mid \hat{R}\right\rangle\right\rangle_{\omega}+(\omega+\varepsilon)\left\langle\left\langle\hat{B}_{-k}^{\dagger} \mid \hat{R}\right\rangle\right\rangle_{\omega}=\frac{i}{2 \pi}\left\langle\left[\hat{B}_{-k}^{\dagger}, \hat{R}\right]\right\rangle
$$

$\hat{R}$ denotes a suitably chosen operator. The geometric factors in these equations of motion are the same as the ones we encoutered in the RPA approach. The system of equations (12a)-(12d) has the same determinant as the system (6a)-(6d) of Part I, with $S$ replacing $\sigma$. This means that the magnon dispersion law in the LSW approach is given by equations (11)-(14) of Part I, with the substitution $\sigma \rightarrow S$ :

$$
\begin{aligned}
E_{1} & =2 S \sqrt{J_{c}\left(1+\cos c k_{z}\right)+2 J_{2}\left(1+\gamma_{2}\left(\mathbf{k}_{\|}\right)\right)-J_{1}\left(\cos a k_{x}+\cos a k_{y}\right)} \times \\
& \times \sqrt{J_{c}\left(1-\cos c k_{z}\right)+2 J_{2}\left(1-\gamma_{2}\left(\mathbf{k}_{\|}\right)\right)+J_{1}\left(\cos a k_{x}-\cos a k_{y}\right)} \\
E_{2} & =2 S \sqrt{J_{c}\left(1+\cos c k_{z}\right)+2 J_{2}\left(1+\gamma_{2}\left(\mathbf{k}_{\|}\right)\right)+J_{1}\left(\cos a k_{x}+\cos a k_{y}\right)} \times \\
& \times \sqrt{J_{c}\left(1-\cos c k_{z}\right)+2 J_{2}\left(1-\gamma_{2}\left(\mathbf{k}_{\|}\right)\right)-J_{1}\left(\cos a k_{x}-\cos a k_{y}\right)} \\
E_{3} & =-E_{1} \\
E_{4} & =-E_{2}
\end{aligned}
$$

We point out that in the LSW approach magnon dispersion is temperature-independent, while in the RPA theory temperature influences the dispersion through the average magnetization $\sigma$. This fact is of no relevance to our present work because we only consider the absolute zero temperature, but it might be important for some future calculations. We are also interested in the ground state magnetization for the Hamiltonian (11). Just like in the RPA approach, we assume that the average magnetization is the same for all four sublattices, so we will only calculate the magnetization for sublattice $A$. From the DM reperesentation one can see that the magnetization (the average value of the operator $\hat{S}^{z}$ ) is given by:

$$
\left\langle\hat{S}_{n}^{z}(A)\right\rangle=S-\left\langle\hat{A}_{n}^{\dagger} \hat{A}_{n}\right\rangle=S-\frac{1}{N} \sum_{k}\left\langle\hat{A}_{k}^{\dagger} \hat{A}_{k}\right\rangle
$$

We use the system of equations (12a)-(12d) (with the choice $\hat{R}=\hat{A}_{k}^{\dagger}$ ) and the spectral theorem to find the correlation function $\left\langle\hat{A}_{k}^{\dagger} \hat{A}_{k}\right\rangle$. We will not give any details here, because the calculations involved are exactly the same as the ones we went through in the RPA approach of Part I. We only give the final result:

$$
\begin{gathered}
\sigma_{S}(0)=S+\frac{1}{2}-\frac{1}{4} I \\
I=\frac{4}{\pi^{3}} \int_{0}^{\pi / 2} d x \int_{0}^{\pi} d y \int_{0}^{\pi / 2} d z\left(\frac{Y+2-X \cos y}{\sqrt{\cdots}_{1}}+\frac{Y+2+X \cos y}{{ }_{\cdots}}\right) \\
\sqrt{\cdots_{1}}=\sqrt{Y(1-\cos z)+X(\cos x-\cos y)+2\left(1-\gamma_{2}\left(\mathbf{k}_{||}\right)\right)} \times \\
\times \sqrt{Y(1+\cos z)-X(\cos x+\cos y)+2\left(1+\gamma_{2}\left(\mathbf{k}_{||}\right)\right)} \\
\sqrt{\cdots_{2}}=\sqrt{Y(1-\cos z)-X\left(\cos a k_{x}-\cos y\right)+2\left(1-\gamma_{2}\left(\mathbf{k}_{\| \mid}\right)\right)} \times \\
\times \sqrt{Y(1+\cos z)+X(\cos x+\cos y)+2\left(1+\gamma_{2}\left(\mathbf{k}_{||}\right)\right)}
\end{gathered}
$$




\section{Numerical calculations and comparison with experiments}

In Figure 1 we give the magnetization as a function of the frustration parameter $X$ in RPA and LSW approaches. The two theories apparently give similar results. However, the roles of quantum fluctuations and interplanar coupling seem to be very different in each of these two theories. This is most easily seen for the $S=1 / 2$ curve. In the LSW theory, the magnetization curve for $S=1 / 2$ drops down rapidly as $|X|$ approaches 2 .
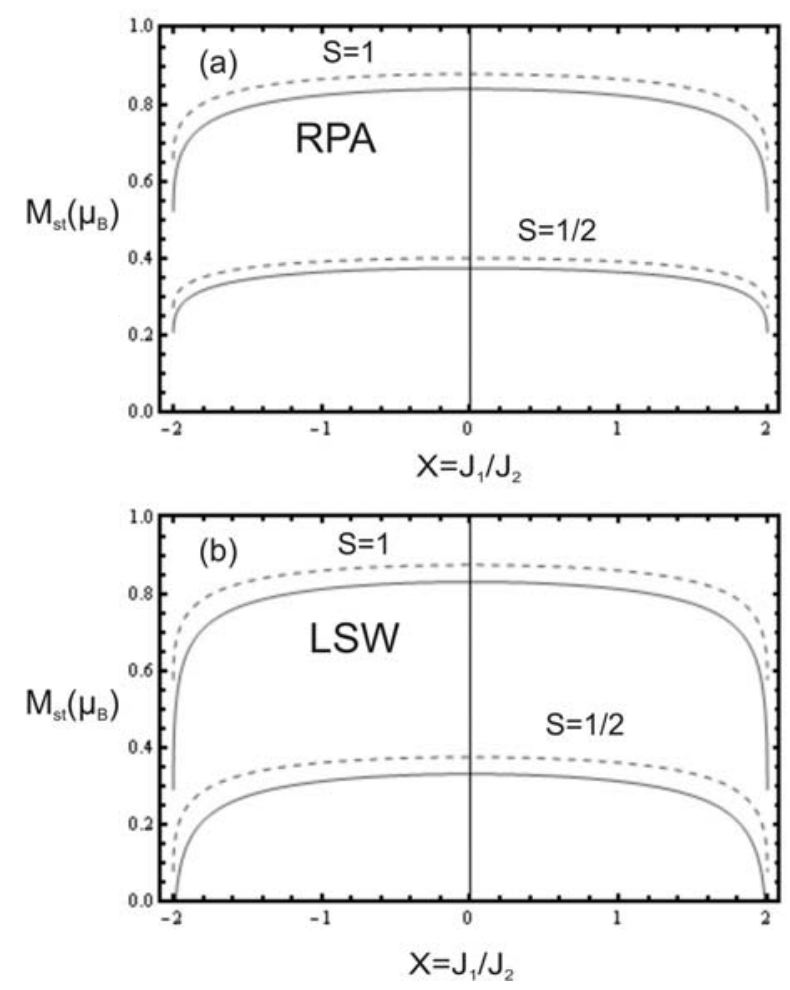

Figure 1. Magnetization as a function of the frustration parameter $X=J_{1} / J_{2}$ in the RPA (a) and LSW (b) approach. Full-line curves correspond to the value of the parameter $Y=0.01$, dashed-line curves correspond to the value $Y=0.1$.

On the other hand, in RPA theory the curve for $S=1 / 2$ deviates only slightly from the main plateau even when $|X|$ takes on values very close to 2 . Also, in the LSW approach the interplanar coupling plays a more significant role in the critical region (region of $|X| \approx 2$ ), because the curves for $Y=0.1$ drop down from the plateau much less than the curves for $Y=0.01$ do. In RPA, the interplanar coupling seems to have little influence on the behavior of staggered magnetization in the critical region.

The fact that magnetization curves in the LSW theory have significantly lower endpoints enables us, in principle, to obtain theoretically the values for observed magnetic momenta in the 1111 type compounds (see Table 1 of Part I). However, in the critical region the magnetization changes rapidly with the frustration parameter $X$, so in order to get the measured values for magnetic momenta in 1111 type compounds agressive fine-tuning would have to be used. This is why we do not consider the LSW results for staggered magnetization to be any better than the RPA results. We are however still left with magnon dispersion as a test of the LSW approach. 
In Figure 2 we show the graphs for the dispersion branches $E_{1}$ and $E_{2}$ in the LSW theory. By comparing them with the corresponding graphs of RPA theory (Figure 3 of Part I) one can see that the two approaches again produce similar results. In fact, apart from slightly higher energy scales in LSW theory, the only noticeable difference comes from the $(0,0,0)-(\pi, 0,0)$ region for the branch $E_{2}$. In RPA, there is little difference in this region between the system deep in the spin strip phase $(X=1)$ and the system close to a quantum phase transition $(X=1.98)$. In LSW, however, the difference between these two phases is quite significant.
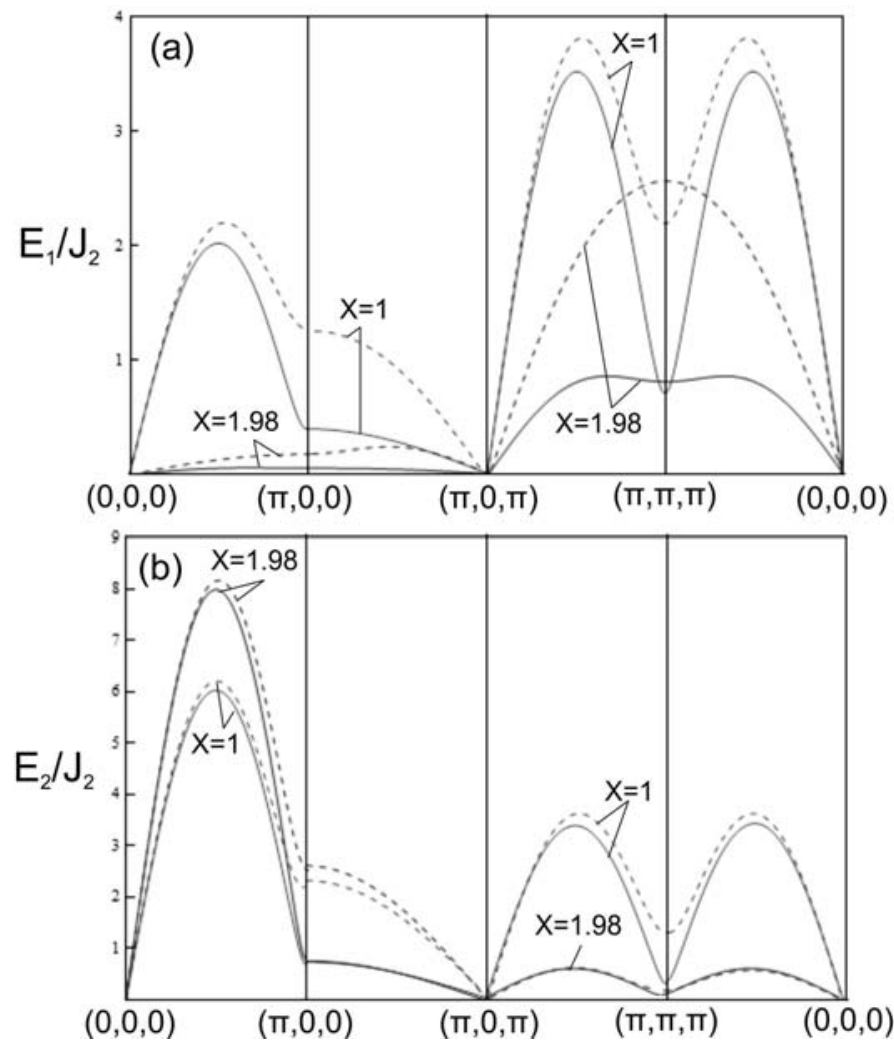

Figure 2. Magnon dispersion branches $E_{1}$ and $E_{2}$ in LSW theory. Full-line curves correspond to the value of the parameter $Y=0.01$, dashed-line curves correspond to the value $Y=0.1$.

A procedure for calculating the velocities of spin waves along crystal axes was explained in some detail in Part I. We could go through this procedure again, but there is a much easier way for us to obtain the velocities of Goldstone mode excitations. Since the RPA dispersion becomes identical with the LSW dispersion after substitution $\sigma \rightarrow S$, we can simply take the equations (39)-(44) of Part I and after carrying out the mentioned substitution we get for the branch $E_{1}$ :

$$
\begin{aligned}
& \frac{v_{x}}{J_{2}}=2 S \sqrt{(2-X)(2-X+Y)} \\
& \frac{v_{y}}{J_{2}}=2 S \sqrt{(2+X)(2-X+Y)} \\
& \frac{v_{z}}{J_{2}}=2 S \sqrt{Y(2-X+Y)}
\end{aligned}
$$


And similarly for the branch $E_{2}$ :

$$
\begin{aligned}
& \frac{v_{x}}{J_{2}}=2 S \sqrt{(2+X)(2+X+Y)} \\
& \frac{v_{y}}{J_{2}}=2 S \sqrt{(2-X)(2+X+Y)} \\
& \frac{v_{x}}{J_{2}}=2 S \sqrt{Y(2+X+Y)}
\end{aligned}
$$

The graphs of spin wave velocities for both branches are given in Figure 3.
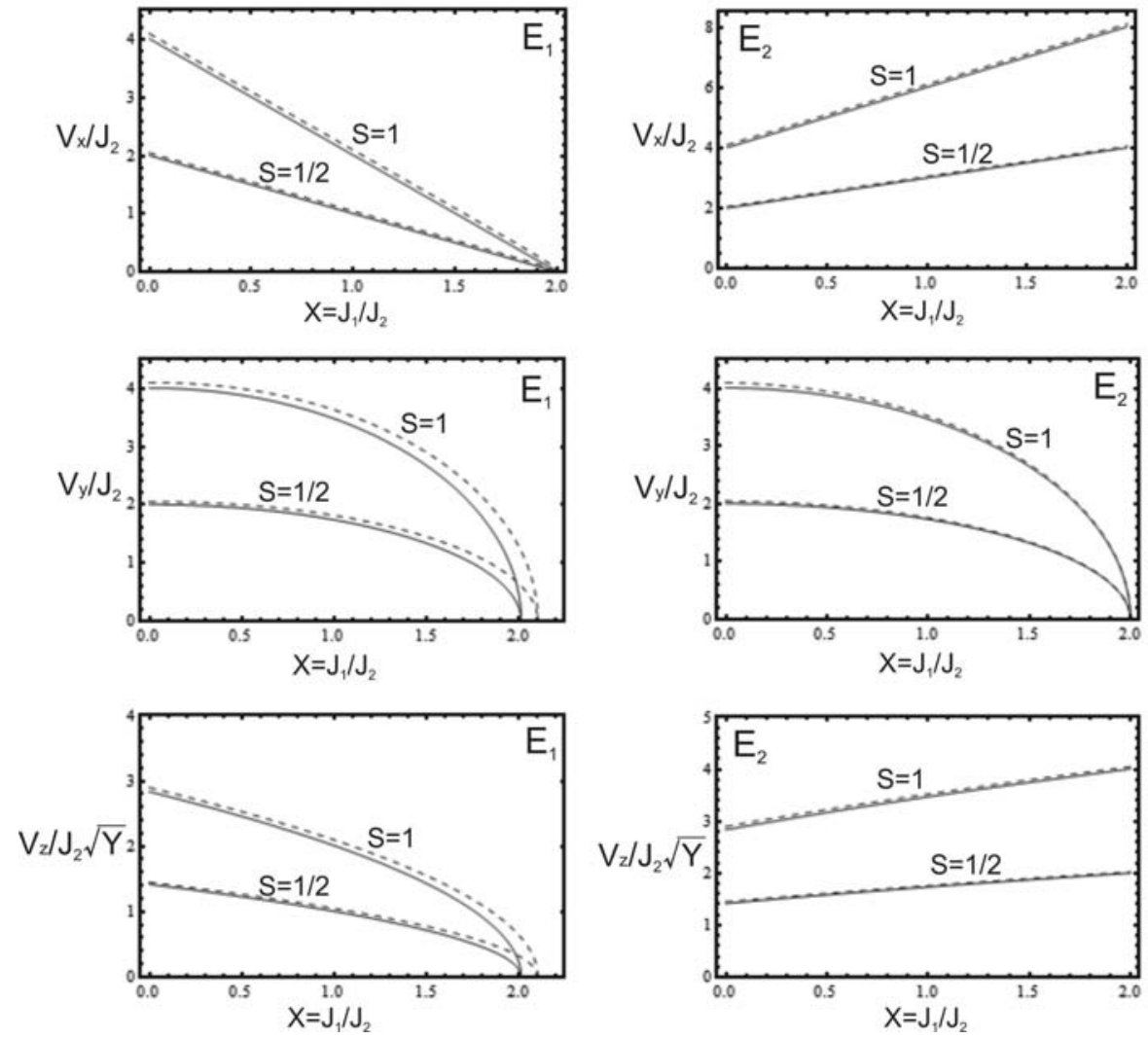

Figure 3. Velocities of spin waves along crystal axes for the branches $E_{1}$ (left) and $E_{2}$ (right). Full-line curves correspond to the value of the parameter $Y=0.01$, dashed-line curves correspond to the value $Y=0.1$.

We mentioned in Part I that there are two kinds of instabilities of the spin stripe order in three dimensions. For a small interplanar coupling (small $Y$ ) magnetization goes to 0 when $|X| \rightarrow 2$, so that there is no long-range order for the critical value of $X$. On the other hand, when $Y$ is large enough the magnetization stays finite when $|X|=2$, but from eqs. (22)-(27) we see that in LSW theory (just like in the RPA theory) one of the spin wave velocities has to vanish when $|X|=2$. This means that the long-range order survives, but not in the form of spin stripes. So, both RPA and LSW approaches allow for two kinds of instabilities of the spin stripe phase. However, the critical value of $Y$ which separates these two kinds of instabilities seems to be very different in RPA and LSW formalisms. 
In this paper we will not estimate the critical values of $Y$, but the results shown in Figure 1 are enough to give us a qualitative overview. In RPA, none of the magnetization curves goes to 0 when $|X|=2$. Even the curve for $S=1 / 2$ and $Y=0.01$ has endpoints which are relatively high. In the LSW theory the situation is very different, and it is evident from Figure 1 that in the LSW approach one would have to choose considerably higher values for $Y$ to keep the magnetization finite than in the RPA formalism.

We are again mostly interested in the ratio $v_{y} / v_{x}$ because it enables us to evaluate the frustration parameter $X$ from the comparison with experimental data. From equations (26) and (25) we get for the branch $E_{2}$ :

$$
\frac{v_{y}}{v_{x}}=\sqrt{\frac{2-X}{2+X}}
$$

This is identical with the result of the RPA approach (eq. (45) of Part I). Again, for the branch $E_{1}$ a reciprocal relation holds. We already mentioned in Part I that the spin wave velocities $v_{x}[9]$ and $v_{z}[10]$ have been estimated with great certainty, but for the velocity $v_{y}$ there is no consesus as nuclear magnetic resonance [11] and neutron diffraction experiments [12] lead to completely different results. That is why we consider two scenarios. From the NMR results one gets $v_{y} / v_{x} \approx 0.1$, and from (28) one finds $X=1.96$. Thus the NMR results imply that iron pnictides are very close to a quantum phase transition. On the other hand, neutron diffraction experiments state that the ratio $v_{y} / v_{x}$ is very close to 0.7 , and from (28) one finds $X=0.7$. Thus according to neutron diffraction results, iron pnictides are deep in the collumnar spin stripe phase, which is in agreement with band structure calculations $[13,14]$. In Figure 4 we give the plots of magnon dispersion for these two scenarios, for both theories (RPA and LSW).
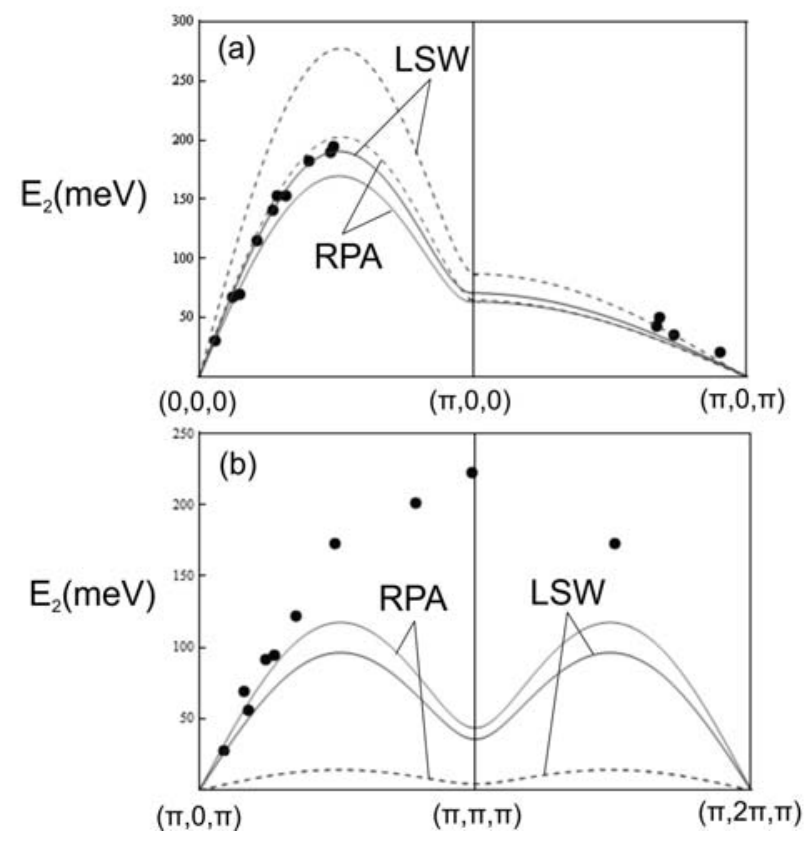

Figure 4. Comparison between the theory and experiment for spin wave dispersion. Full-line curves correspond to the value of the parameter $X=0.7$, dashed-line curves correspond to the value $X=1.96$. All theoretical results are for $Y=0.1$. 
Unfortunately, we see that the LSW theory brings little to no improvement over the RPA results. The LSW curve for $X=0.7$ fits the experimental data almost perfectly in the region $(0,0,0)-(\pi, 0,0)$. On the other hand, the LSW curve for $X=0.1$ is the one which deviates mostly from experimental results in the same region. As for the region $(\pi, 0, \pi)-(\pi, 2 \pi, \pi)$, the LSW curve for $X=0.7$ gives an even worse estimate for experimental data than the corresponding RPA curve. Since both RPA and LSW approaches fail to provide a completely satisfactory description of the magnetic properties of iron pnictides, we doubt that keeping the $\hat{H}_{4}$ term in the model Hamiltonian (8) would lead to significant (if any) improvements. The problem therefore seems to be in the original model Hamiltonian (1), which needs to be modified to give better theoretical results. Some papers mention the possibility that an almost perfect agreement between theory and experiment can be achieved if spatially anisotropic Hamiltonian is used, with different NN exchange integrals along $x$ and $y$ axes (see, for example, the discussion in Ref. [12]). However, if a spatially anisotropic Hamiltonian is used then there arises the question of the physical origin of this anisotropy. The orthorhombic or monoclinic distortions are much too small to account for great differences in the NN exchange integrals needed to fit the experimental data. Some authors have suggested that the magnetic order itself might be responsible for this anisotropy, if the biquadratic exchange interaction is strong enough in iron pnictides [15].

\section{Conclusion}

We attempted to decribe the magnetic properties of parent pnictide compounds by using the isotropic $J_{1}-J_{2}-J_{c}$ Heisenberg model Hamiltonian. We used two different formalisms - the spin formalism in random phase approximation and the boson formalism in Bloch's approximation, or linear spin wave theory. We compared the numerical calculations for ground state magnetization and magnon dispersion for these two approaches and concluded that they give very similar results. It seems virtually impossible to distinguish between the results of these two theories experimentally. Unfortunately, both approaches give inadequate results for local magnetic momenta in 1111 type compounds and for magnon dispersion in the $(\pi, 0, \pi)-(\pi, 2 \pi, \pi)$ region. We conclude that the model Hamiltonian itself is not completely suitable for the parent pnictide compounds and that it needs to be modified. These modifications might include an introduction of the anisotropic nearest-neighbor interactions and will be considered in future publications.

\section{Acknowledgments}

This work was supported by the Serbian Ministry of Education and Science, Project No. OI 171009.

\section{References}

[1] M. Vujinović, M. Pantić, D. Kapor, S. Radošević, J. Res. Phys. 35, 29 (2011)

[2] S. V. Tyablikov, The Methods in the Quantum Theory of Magnetism, (Plenum Press, 1967). 
[3] W. Nolting, A. Ramakanth, Quantum Theory of Magnetism, (Springer-Verlag, Berlin Heidelberg, 2009).

[4] B. S. Tošić, Statistička fizika, (PMF u Novom Sadu, Novi Sad, 1978),

M. Pantić, Predavanja iz statističke fizike (lecture notes), Novi Sad, 2009

[5] F. J. Dyson, Phys. Rev. 102, 1217 (1956).

[6] S. V. Maljejev, Zh. Ekspr. Teor. Fiz. 33, 1010 (1957).

[7] Y. Izyumov, E. Kurmaev, High-Tc. Superconductors Based on FeAs Compounds, (Springer-Verlag, Berlin Heidelberg, 2010).

[8] M. M. Korshunov, I. Eremin, Phys. Rev. B 78, 140509(R) (2008).

[9] J. Zhao, D.-X. Yao, S. Li, T. Hong, Y. Chen, S. Chang, W. R. II, J. W. Lynn, H. A. Mook, G. F. Chen et al., Phys. Rev. Lett. 101, 167203 (2008).

[10] R. A. Ewings, T. G. Perring, R. I. Bewley, T. Guidi, M. J. Pitcher, D. R. Parker, S. J. Clarke, and A. T. Boothroyd, Phys. Rev. B 78, 220501(R) (2008).

[11] A. Ong, G. S. Uhrig, O. P. Sushkov, Phys. Rev. B 80, 014514 (2009).

[12] J. Zhao, D. T. Adroja, D.-X. Yao, R. Bewley, S. Li, X. F. Wang, G. Wu, X. H. Chen, J. Hu, P. Dai, Nat. Phys. 5, 555 (2009).

[13] Z. P. Yin, S. Lebegue, M. J. Han, B. P. Neal, S. Y. Savrasov, and W. E. Pickett, Phys. Rev. Lett. 101, 047001 (2008).

[14] C. Cao, P. J. Hirschfeld, and H.-P. Cheng, Phys. Rev. B 77, 220506 (2008).

[15] D. Stanek, O. P. Sushkov, and G. S. Uhrig, arXiv:1104.1954v1 (2011). 